\title{
InCONSISTENT MODELS FOR RELEVANT ARITHMETICS
}

\author{
Robert K. Meyer and Chris Mortensen \\ Australian National University \\ University of Adelaide
}

\section{Introduction}

This paper develops in certain directions the work of Meyer in [3], [4], [5] and [6] (see also Routley [10] and Asenjo [11]). In those works, Peano's axioms for arithmetic were formulated with a logical base of the relevant logic R, and it was proved finitistically that the resulting arithmetic, called $R^{\sharp}$, was absolutely consistent. It was pointed out that such a result escapes incautious formulations of Gödel's second incompleteness theorem, and provides a basis for a revived Hilbert programme. The absolute consistency result used as a model arithmetic modulo two. Modulo arithmetics are not ordinarily thought of as an extension of Peano arithmetic, since some of the propositions of the latter, such as that zero is the successor of no number, fail in the former. Consequently a logical base which, unlike classical logic, tolerates contradictory theories was used for the model. The logical base for the model was the three-valued logic RM3 (see e.g. [1] or [8]), which has the advantage that while it is an extension of $R$, it is finite valued and so easier to handle.

The resulting model-theoretic structure (called in this paper $\mathrm{RM}^{2}$ ) is interesting in its own right in that the set of sentences true therein constitutes a negation inconsistent but absolutely consistent arithmetic which is an extension of $R^{\sharp}$. In fact, in the light of the result of [6], it is an extension of Peano arithmetic with a base of a classical logic, $P^{\sharp}$. A generalisation of the structure is to modulo arithmetics with the same logical base RM3,

Australasian Journal of Logic (18:5) 2021, Article no. 6 
but with varying moduli (called $\mathrm{RM}^{i}$ here). We first study the properties of these arithmetics in this paper. The study is then generalised by varying the logical base, to give the arithmetics $\mathrm{RMn}^{i}$, of logical base $\mathrm{RMn}$ and modulus $i$. Not all of these exist, however, as arithmetical properties and logical properties interact, as we will show. The arithmetics $\mathrm{RMn}^{i}$ give rise, on intersection, to an inconsistent arithmetic $\mathrm{RM}^{\omega}$ which is not of modulo $i$ for any $i$. We also study its properties, and, among other results, we show by finitistic means that the more natural relevant arithmetics $R^{\sharp}$ and $R^{\sharp \sharp}$ are incomplete (whether or not consistent and recursively enumerable). In the rest of the paper we apply these techniques to several topics, particularly relevant quantum arithmetic in which we are able to show (unlike classical quantum arithmetic) that the law of distribution remains unprovable. Aside from its intrinsic interest, we regard the present exercise as a demonstration that inconsistent theories and models are of mathematical worth and interest.

\section{Definitions}

We begin with an arithmetical language $\mathscr{L}$ with a single binary relation $=$, constant 0 , variables $x, y, z, \ldots$, connectives $\neg, \&, \rightarrow$, term operators,$+ \times$, ', and universal quantifier ( ) (also written $(\forall)$ ). Terms (open and closed) and sentences and wffs (open and closed) are defined in the usual way. ${ }^{1} \exists$ is defined as $\neg \forall \neg, A \vee B$ is defined as $\neg(\neg A \& \neg B)$, and $0^{(n)}$ is an abbreviation for $0^{\prime \prime} \ldots{ }^{\prime}$, with $n$ iterations of the superscript.

Definition 1. The relevant logic $\mathrm{RQ}$ is given by the following axiom schemata and rules. Axioms:

(1) $\quad(A \rightarrow B) \rightarrow((B \rightarrow C) \rightarrow(A \rightarrow C))$

(2) $\quad A \rightarrow((A \rightarrow B) \rightarrow B)$

(3) $\quad A \& B \rightarrow A$

(4) $\quad A \& B \rightarrow B$

(5) $(A \rightarrow B) \&(A \rightarrow C) \rightarrow(A \rightarrow B \& C)$

(6) $\quad A \rightarrow A \vee B$

(7) $B \rightarrow A \vee B$

(8) $(A \rightarrow C) \&(B \rightarrow C) \rightarrow(A \vee B \rightarrow C)$

${ }^{1}$ We occasionally use $F x_{1} \ldots x_{n}$ for an open formula in which $x_{1} \ldots x_{n}$ may occur free.

Australasian Journal of Logic (18:5) 2021, Article no. 6 
(9) $\quad A \&(B \vee C) \rightarrow(A \& B) \vee(A \& C)$

(10) $\neg \neg A \rightarrow A$

(11) $\quad(A \rightarrow \neg A) \rightarrow \neg A$

and the following quantificational schemata and all closures of them:

(12) $(x) A \rightarrow(t / x)(t$ any term)

(13) $\quad(x)(A \rightarrow B) \rightarrow((x) A \rightarrow(x) B$

(14) $A \rightarrow(x) A(x$ not free in $A)$

(15) $(x)(A \vee B) \rightarrow(A \vee(x) B)(x$ not free in $A)$

(16) $\quad(x) A \&(x) B \rightarrow(x)(A \& B)$

Rules:

(17) $\vdash A, \vdash A \rightarrow B / \therefore \vdash B$

(18) $\vdash A, \vdash B / \therefore \vdash A \& B$

To obtain the logic RMQ, add the Mingle axiom $A \rightarrow(A \rightarrow A)$. The relevant arithmetics $\mathrm{R}^{\sharp}$ and $\mathrm{RM}^{\sharp}$ in the language of $\mathscr{L}$ are given by the following. Logical axioms and rules are those of $\mathrm{R}$ and $\mathrm{RM}$ respectively. Both have additional artihmetical axioms and rules:

Arithmetical axioms:

$$
\begin{array}{ll}
\# 1 & (x, y)\left(x=y \leftrightarrow x^{\prime}=y^{\prime}\right) \\
\# 2 & (x, y, z)(x=y \rightarrow(x=z \rightarrow y=z)) \\
\# 3 & (x)\left(x^{\prime} \neq 0\right) \\
\# 4 & (x)(x+0=x) \\
\# 5 & (x, y)\left(x+y^{\prime}=(x+y)^{\prime}\right) \\
\# 6 & (x)(x \times 0=0) \\
\# 7 & (x, y)\left(x \times y^{\prime}=(x \times y)+x\right)
\end{array}
$$

Arithmetical rule:

$\mathrm{RMI} \quad \vdash F 0, \vdash(x)\left(F x \rightarrow F x^{\prime}\right) / \therefore \vdash(x) F x$

Australasian Journal of Logic (18:5) 2021, Article no. 6 
To obtain the arithmetics $\mathrm{R}^{\sharp \sharp}$ and $\mathrm{RM}^{\sharp \sharp}$, add to $\mathrm{R}^{\sharp}$ and $\mathrm{RM}^{\sharp}$ respectively the rule

$$
\Omega \vdash F 0, \vdash F 0^{\prime}, \ldots, \vdash F 0^{(n)}, \ldots(\text { for all } n) / \therefore \vdash(x) F x
$$

The arithmetics $\mathrm{P}^{\sharp}$ and $\mathrm{P}^{\sharp}$ are Peano arithmetic with a base of classical logic and, respectively, without and with rule $\Omega$.

Definition 2. An $\mathrm{RM}^{i}$ model is an ordered pair $\left\langle D^{i}, I\right\rangle$ where $D^{i}$ are the integers modulo $i$, and $I$ is a function which assigns to the terms, operators and wffs of $\mathscr{L}$ the following values.

(1) For individual variables $x, I(x) \in D^{i}$

(2) $I(0)=0$

(3) $I(+), I(\times), I\left(^{\prime}\right)$ are the operations,$+ \times,{ }^{\prime}$ of arithmetic modulo $i$ respectively.

(4) For terms $t_{1}, t_{2}, I\left(t_{1}+t_{2}=I(+)\left(I\left(t_{1}\right), I\left(t_{2}\right)\right), I\left(t_{1} \times t_{2}=\right.\right.$ $I(\times)\left(I\left(t_{1}\right), I\left(t_{2}\right)\right), I\left(t_{1}^{\prime}\right)=I\left(^{\prime}\right) I\left(t_{1}\right)$

Open and closed wffs are assigned values in the three-valued Sugihara matrix $\{+1,0,-1\}$ characteristic for RM3 as follows.

(5) For atomic wffs $t_{1}=t_{2}$, where $t_{1}, t_{2}$ are terms, $I\left(t_{1}=t_{2}\right)=0$ iff $I\left(t_{1}\right)=I\left(t_{2}\right)$ (modulo $i$ ), otherwise $I\left(t_{1}=t_{2}\right)=-1$.

(6) $I(A \rightarrow B), I(A \& B)$ and $I(\neg A))$ are determined by the three-valued tables for RM3.

(7) $I((x) A)=\operatorname{glb}\left\{y: I^{\prime}(A)=y\right\}$ for every $x$-variant $I^{\prime}$ of $I$.

A sentence $A$ is $\mathrm{RM}^{i}$-true under interpretation $I$ iff $I(A) \in\{+1,0\} . A$ is true in the model $\mathrm{RM}^{i}$ iff $A$ is $\mathrm{RM}^{i}$-true under all interpretations $I$. The arithmetic $\mathrm{RM}^{i}$ is the set of sentences true in the model $\mathrm{RM}^{i}$.

Definition 3. A subset $S$ of $\mathscr{L}$ is an L-theory (relative to logic L) iff (1) if $A \in S$ and $\vdash_{\mathrm{L}} A \rightarrow B$ then $B \in S$, and (2) if $A \in S$ and $B \in S$ then $A \& B \in S$. An L-theory $S$ is (negation) consistent iff for all closed wffs $A \in \mathscr{L}$, not both $A \in S$ and $\neg A \in S$; otherwise inconsistent. $S$ is trivial (or absolutely inconsistent) iff $S=\mathscr{L}$, otherwise nontrivial. $S$ is $\omega$-inconsistent iff for some $F, F 0^{(n)} \in S$ for every $n$, and also $\neg(x) F x \in S$. S is complete

Australasian Journal of Logic (18:5) 2021, Article no. 6 
iff for all closed wffs $A$, either $A \in S$ or $\neg A \in S$. $S$ is $\omega$-complete iff the rule $\Omega$ holds for $S$, i.e. for all $F$, if $F O^{(n)} \in S$ for every $n$, then $(x) F x \in S$. The rule $\gamma$ holds for $S$ iff, if $A \in S$ and $\neg A \vee B \in S$, then $B \in S$. $S$ is prime iff for all closed wffs $A, B$ if $A \vee B \in S$ then at least one of $A \in S, B \in S$

\section{The Arithmetics $\mathrm{RM}^{i}$}

Proposition 1. The arithmetic $\mathrm{RM} 3^{2}$ is an $\mathrm{RQ}$ theory. Every theorem of $\mathrm{R}^{\sharp}$ is true in the model $\mathrm{RM}^{2}$. The wff $0=0^{\prime}$ is not true in that model. Hence $\mathrm{R}^{\sharp}$ is absolutely consistent.

Proof. See [3].

The significance of this result is, as discussed in [3], [4] and [5], that relevant Peano arithmetic can be finitistically proved to be absolutely consistent, unlike classical Peano arithmetic $P^{\sharp}$. This goes some way to resurrecting the programme of Hilbert of a finitistic demonstration of the (absolute) consistency of mathematics. However, every primitive recursive function is representable in $R^{\sharp}$, so a proof of the negation consistency of $R^{\sharp}$ within $R^{\sharp}$ remains impossible, by Gödel's second incompleteness theorem. It is also provable that $P^{\sharp \sharp}$ is included in $R^{\sharp \sharp ~(s e e ~[6]) ~ s o ~ t h a t, ~ s i n c e ~} P^{\sharp} \subset P^{\sharp \sharp}$ and $R^{\sharp \sharp} \subset R M 3^{2}$, we do have a proof of the absolute consistency of $P^{\sharp}$ (and hence of its negation consistency, which coincides with absolute consistency in $P^{\sharp}$ but not $R^{\sharp \sharp}$ ). This does not run contrary to Gödel's second theorem, however, since one step in the proof, viz. $P^{\sharp \sharp} \subset R^{\sharp \sharp}$, uses methods not representable in $R^{\sharp}$. Again, the negation consistency of $R^{\sharp \sharp ~(a n d ~ h e n c e ~} R^{\sharp}$ ) is demonstrable (see [6]), but the result uses equally strong methods. These results also constitute an argument for the pragmatic virtues of the relevant logics. In fact, as we show later by finitistic methods, no sentence of the form $0^{(n)}=0^{(m)}$ for distinct $n$, $m$ is provable in any of $R^{\sharp}, R^{\sharp \sharp}, R M^{\sharp}, R M^{\sharp \sharp}$. (See also [3].) Hence relevant logic has considerably better control over its Peano arithmetic than does classical logic. We go on now to consider the properties of the arithmetics RM3 ${ }^{i}$.

Proposition 2. Every arithmetic $\mathrm{RM}^{i}$ is inconsistent and $\omega$-inconsistent but absolutely consistent.

Proof. $0=0^{\prime}$ is easily seen not to be in any $\mathrm{RM}^{i}$, so all are absolutely consistent. But all are inconsistent, since (e.g.) in any model $\mathrm{RM}^{i} I(0=$ $0)=0$, so by the RM3 tables for negation, $I(\neg(0=0))=0$ also, hence

Australasian Journal of Logic (18:5) 2021, Article no. 6 
both are true. For $\omega$-inconsistency, observe that any sentence of the form $\neg\left(0^{(n)}=0^{(m)}\right)$ takes value +1 or 0 in every RM3 ${ }^{i}$. So in particular $\neg(0=0)$, $\neg\left(0=0^{\prime}\right), \ldots, \neg\left(0=0^{(n)}\right), \ldots$ are all theorems. But the value of $\neg(0=0)$ is 0 , so $(x) \neg(0=x)$ takes a maximum value of 0 , so $\neg(x) \neg(0=x)$ takes a minimum value of 0 (by tables for $\neg$ ), and so is a theorem.

Proposition 3. For any $F, \vdash_{\mathrm{RM}^{i}}\left(F 0 \& \ldots \& F 0^{(i-1)}\right) \rightarrow(x) F x$.

Proof. If $F 0 \& \ldots \& F 0^{(i-1)}$ takes the value +1 , then by all the RM3 tables for $\rightarrow$, each conjunct does. Any assignment to the variable in $F x$ must therefore also give the value +1 , whence $(x) F x$ takes that value. If the antecedent takes the value 0 , each conjunct takes the value 0 or +1 , so again any assignment to the variable in $F x$ takes value 0 or +1 (and 0 for some assignment). Hence $(x) F x$ takes value 0 , and so by the RM3 tables, $\left(F 0 \& \ldots \& F 0^{(i-1)}\right) \rightarrow(x) F x$ takes value 0 . If the antecedent takes value -1 , the whole wff takes value +1 .

Proposition 4. $\mathrm{RM}^{i}$ is $\omega$-complete.

Proof. If $\vdash F 0, \ldots, \vdash F 0^{(n)}, \ldots$ for every $n$, then $\vdash F 0, \ldots, \vdash F 0^{(i-1)}$; so that $\vdash F 0 \& \ldots \& F 0^{(i-1)}$. Hence by Proposition 3 and the fact that each $\mathrm{RM}^{i}$ is closed under modus ponens for $\rightarrow, \vdash(x) F x$.

Proposition 5. $\mathrm{RM}^{i}$ contains $\mathrm{R}^{\sharp}, \mathrm{R}^{\sharp}, \mathrm{RM}^{\sharp}, \mathrm{RM}^{\sharp \sharp}$.

Proof. As in [2], this is a matter of verifying that the axioms of $\mathrm{R}^{\sharp}$ etc. are true in $\mathrm{RM}^{i}$ and that the rules preserve truth. That $\Omega$ is truth preserving follows from Proposition 4.

Proposition 6. $\mathrm{RM}^{i}$ is complete and prime.

Proof. Primeness follows from the RM3 tables for $\vee$ : for closed wffs $A, B$, if $I(A \vee B) \in\{+1,0\}$ one of $I(A), I(B)$ must do so. Completeness is then immediate on the observation, easily checked, that $\vdash_{\mathrm{RM}^{i}} A \vee \neg A$.

Proposition 7. $\mathrm{RM}^{i}$ is decidable.

Proof. We observe first that it is standard to show that an open wff $A$ is a theorem iff its universal closure is. By Proposition 3, however, all universal quantifiers in closed wffs are eliminable in favour of finite conjunctions. Since the value of atomic wffs $0^{(n)}=0^{(m)}$ is effectively given, the value of all universally closed formulas, conjunctions and negations can be calculated.

Australasian Journal of Logic (18:5) 2021, Article no. 6 


\section{Axiomatising $\mathrm{RM}^{i}$}

The purpose of this section is to axiomatise the arithmetics $\mathrm{RM}^{i}$. We proceed via a number of lemmas.

Definition 4. $\mathrm{RM}^{i \sharp}$ is the arithmetic obtained by adding to $\mathrm{RM}^{\sharp}$ the axioms $0=0^{(i)}$, and for every $j, 2 \leq j<i, 0=0^{(j)} \leftrightarrow 0=0^{\prime}$.

Lemma 1. In $\mathrm{RM}^{i \sharp}, \vdash\left(x, y, z_{1}, \ldots, z_{n}\right)\left(x=y \& F x z_{1} \ldots z_{n} \rightarrow F y z_{1} \ldots z_{n}\right)$.

Proof. In [4] or [5].

Lemma 2. In $\mathrm{RM}^{i \sharp}, \vdash(x)\left(x=0 \vee x=0^{\prime} \vee \ldots \vee x=0^{(i-1)}\right)$.

Proof. The proof uses the rule $R M I$ (Definition 1). Plainly we have $\vdash 0=$ $0 \vee 0=0^{\prime} \vee \ldots \vee 0=0^{(i-1)}$. But also we have, from axiom $\# 1, \vdash(x)(x=$ $\left.0 \rightarrow x^{\prime}=0^{\prime}\right), \vdash(x)\left(x=0^{\prime} \rightarrow x^{\prime}=0^{(2)}\right), \ldots, \vdash(x)\left(x=0^{(i-2)} \rightarrow x^{\prime}=0^{(i-1)}\right)$. Also, using $0=0^{(i)}$ and Lemma $1, \vdash(x)\left(x=0^{(i-1)} \rightarrow x^{\prime}=0\right)$. Hence by $\mathrm{RMQ}$ principles, $\vdash(x)\left(x=0 \vee \ldots \vee x=0^{(i-1)} \rightarrow x^{\prime}=0 \vee \ldots \vee x^{\prime}=0^{(i-1)}\right)$. The lemma follows using rule $R M I$.

Lemma 3. In $\mathrm{RM}^{i \sharp}, \vdash\left(F 0 \& \ldots \& F 0^{(i-1)}\right) \leftrightarrow(x) F x$, if $x$ is not free in $F$.

Proof. Right to left is immediate from axiom (12). Left to right: using Lemma 1 and RMQ principles, we have

$$
\vdash(x)\left(\left(x=0 \vee \ldots \vee x=0^{(i-1)}\right) \& F 0 \& \ldots \& F 0^{(i-1)} \rightarrow F x\right) .
$$

Distributing the quantifier,

$$
\vdash(x)\left(x=0 \vee \ldots \vee x=0^{(i-1)}\right) \&(x)\left(F 0 \& \ldots \& F 0^{(i-1)}\right) \rightarrow(x) F x .
$$

The first conjunct of the antecedent is a theorem. Hence if $x$ is not free in $F$, we have from axiom (14) and rule (18), the derived rule

$$
D: \quad \vdash F 0, \ldots, \vdash F 0^{(i-1)} / \therefore \vdash(x) F x
$$

But now we also have $\vdash F 0 \& \ldots \& F 0^{(i-1)} \rightarrow F 0, \ldots, \vdash F 0 \& \ldots \& F 0^{(i-1)} \rightarrow$ $F 0^{(i-1)}$. Hence by rule $D, \vdash(x)\left(F 0 \& \ldots \& F 0^{(i-1)} \rightarrow F x\right)$. Distributing quantifiers and using axiom (14) we have $\vdash F 0 \& \ldots \& F 0^{(i-1)} \rightarrow(x) F x$ as 
required. ${ }^{2}$

Definition 5. Let $\mathbf{t}$ be $0=0$ and $\mathbf{f}$ be $\neg 0=0$.

Lemma 4. In $\mathrm{RM}^{i \sharp}{ }^{\sharp}, 0=0^{\prime}, \neg 0=0^{\prime}$, $\mathbf{t}$ and $\mathbf{f}$ are related as in the $\mathrm{RM} 3$ three-valued Sugihara chain below, with the $\&$ and $\rightarrow$ relations between them as for RM3.

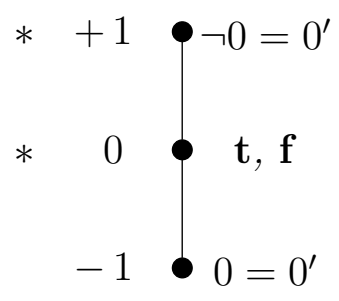

Proof. The proof, essentially verifying theorems in $\mathrm{RM}^{i \sharp}$, is lengthy but not difficult. Details are omitted.

Lemma 5. In $\mathrm{RM}^{i \sharp}$, for any wff $A$, we have either $\vdash A \leftrightarrow 0=0^{\prime}$ or $\vdash A \leftrightarrow$ $0=0$ or $\vdash A \leftrightarrow \neg 0=0^{\prime}$.

Proof. By induction on the complexity of $A$. We need only consider the case where $A$ is closed, since the case where $A$ is open follows by axiom (12). Furthermore the quantifier case in the inductive clause can be ignored since by Lemma 3, quantifiers can be eliminated in favour of a conjunction.

Base. $A$ is $t_{1}=t_{2}$. In view of Lemmas 1 and 2 , we need only consider where $t_{1}=t_{2} \in\left\{0,0^{\prime}, \ldots, 0^{(i-1)}\right\}$. First, let $A$ be $0^{(n)}=0^{(n)}$ for some $n$, $0<n \leq i-1$. Now certainly $\vdash 0=0 \leftrightarrow 0=0$; but also $\vdash 0^{\prime}=0^{\prime} \leftrightarrow 0=0$, $\ldots, \vdash 0^{(i-1)}=0^{(i-1)} \leftrightarrow 0=0$, by repeated applications of axiom $\# 1$. If $A$ is $0^{(n)}=0^{(m)}$ where $n<m \leq i-1$, then we have $\vdash 0=0^{(|n-m|)} \leftrightarrow 0^{\prime}=$ $0^{(|n-m|+1)}, \vdash 0^{\prime}=0^{(|n-m|+1)} \leftrightarrow 0^{(2)}=0^{(|n-m|+2)}, \ldots, \vdash 0^{(n-1)}=0^{(m-1)} \leftrightarrow$ $0^{(n)}=0^{(m)}$, whence $\vdash 0=0^{(|n-m|)} \leftrightarrow 0^{(n)}=0^{(m)}$. But it is an axiom that $\vdash 0=0^{\prime} \leftrightarrow 0=0^{(|n-m|)}$. Hence $\vdash 0=0^{\prime} \leftrightarrow 0^{(n)}=0^{(m)}$. If $n>m$, we have $\vdash 0^{(n)}=0^{(m)} \leftrightarrow 0^{(m)}=0^{(n)}$ via the symmetry of identity (which follows from $\# 2$ ), which reduces the case to the previous one.

\footnotetext{
${ }^{2}$ We also note that it is straightforward to show that provable equivalences are replaceable in all contexts. See [4] or [5].
}

Australasian Journal of Logic (18:5) 2021, Article no. 6 
Inductive clause. (a) Negations. If $\vdash A \leftrightarrow 0=0^{\prime}$ or $\vdash A \leftrightarrow 0=0$ or $\vdash A \leftrightarrow \neg 0=0^{\prime}$, then evidently $\vdash \neg A \leftrightarrow \neg 0=0^{\prime}$ or $\vdash \neg A \leftrightarrow 0=0$ or $\vdash \neg A \leftrightarrow 0=0^{\prime}$. The second disjunct uses Lemma 4. (b) Conjunctions. We have $\vdash A \leftrightarrow 0=0^{\prime}$ or $\vdash A \leftrightarrow 0=0$ or $\vdash A \leftrightarrow \neg 0=0^{\prime}$, and $\vdash B \leftrightarrow 0=0^{\prime}$ or $\vdash B \leftrightarrow 0=0$ or $\vdash B \leftrightarrow \neg 0=0^{\prime}$. We have to show $\vdash A \& B \leftrightarrow 0=0^{\prime}$ or $\vdash A \& B \leftrightarrow 0=0$ or $\vdash A \& B \leftrightarrow \neg 0=0^{\prime}$. Evidently these follow from the equivalences of Lemma 4. (c) Implications. These follow, in a like fashion, from the equivalences of Lemma 4 . This completes the lemma.

Lemma 5 means that adding to $\mathrm{RM}^{\sharp} 0=0^{(i)}$ and $0=0^{(j)} \leftrightarrow 0=1$ for all $j$ with $2 \leq j<i$ forces exactly three distinct propositions on us, two the negations of each other and the third its own negation. But that is precisely the structure of the logic RM3. Thus there would be no point in attempting a weaker logical extension of RM3 with these axions (though we should note that, in general, the $0=0^{(j)} \leftrightarrow 0=0^{\prime}$ are not all independent) since only three of its values would be used, isomorphically with RM3. Lemma 5 also enables us to axiomatize the models $\mathrm{RM}^{i}$.

Proposition 8. The theorems of $\mathrm{RM}^{i \sharp}$ are exactly the truths of $\mathrm{RM}^{i}$.

Proof. That all theorems of $\mathrm{RM}^{i \sharp}$ are truths of $\mathrm{RM}^{i}$ is a standard matter of showing that the (extra) axioms are true, since we already have that $\mathrm{RM}^{\sharp}$ is verified in $\mathrm{RM}^{i}$. Lemma 5 does all the work in the converse. For suppose $A$ is true in $\mathrm{RM}^{i}$. Now certainly not $\vdash A \leftrightarrow 0=0^{\prime}$, for, since all theorems of $\mathrm{RM}^{i \sharp}$ are true, $A \leftrightarrow 0=0^{\prime}$ would be true, so that $0=0^{\prime}$ would be true (by a simple argument). Hence $\vdash A \leftrightarrow \neg 0=0^{\prime}$ or $\vdash A \leftrightarrow 0=0$. But $\vdash \neg 0=0^{\prime}$ and $\vdash 0=0$. Hence $\vdash A$.

\section{Extending the Logic: The Arithmetics $\mathrm{RMn}^{i}$}

When we move to consider weakening the logical base to the logics RMn $(n$ odd: an even $n$ will not permit an inconsistent arithmetic) characterised by the $n$-valued Sugihara matrices or chains, we run into the problem that not every modulus will permit distinct false equations to occupy distinct false points in the matrix, enabling us to take advantage of the logical resources of the weaker logic (longer chain). In fact, we prove that given the logical apparatus of RM, if $j$ and $k$ are relatively prime, then at least one of $0=0^{(j)}$ and $0=0^{(k)}$ is equivalent to $0=0^{\prime}$. To show this, we prove something quite a bit stronger which is of independent interest.

Australasian Journal of Logic (18:5) 2021, Article no. 6 
Proposition 9. In $\mathrm{R}^{\sharp}$, for any $i$, $j$ we have $\vdash\left(0=0^{(i)} \circ 0=0^{(j)}\right) \leftrightarrow 0=$ $0^{(\operatorname{gcd}(i, j))}$, where $A \circ B=_{D F} \neg(A \rightarrow \neg B)$, and $\operatorname{gcd}(i, j)$ is the greatest common divisor of $i$ and $j$.

Proof. Right to left. $\vdash 0=0^{(i)} \rightarrow 0=0^{(k i)}$ (any $k, i$ ). (Reason: $\vdash 0=$ $0^{(i)} \rightarrow 0^{(i)}=0^{(2 i)} \rightarrow 0=0^{(2 i)}$, but $\vdash 0=0^{(i)} \leftrightarrow 0^{(i)}=0^{(2 i)}$; hence, $\vdash 0=0^{(i)} \rightarrow 0=0^{(2 i)}$. Now $\vdash 0=0^{(i)} \rightarrow 0^{(i)}=0^{(3 i)} \rightarrow 0=0^{(3 i)}$. But $\vdash 0^{(i)}=0^{(3 i)} \leftrightarrow 0=0^{(2 i)}$; so from $\vdash 0=0^{(i)} \rightarrow 0=0^{(2 i)}, \vdash 0=0^{(i)} \rightarrow$ $0=0^{(3 i)}$. And so on.) Hence, since $\operatorname{gcd}(i, j)$ divides both $i$ and $j$, we have $\vdash 0=0^{(\operatorname{gcd}(i, j))} \rightarrow 0=0^{(i)} \& 0=0^{(j)}$. But $\vdash_{\mathrm{R}} A \& B \rightarrow A \circ B$.

Left to right. First note that $\vdash_{\mathrm{R}} A \rightarrow(B \rightarrow C) \leftrightarrow$. $(A \circ B) \rightarrow C$, so it suffices to prove $\vdash 0=0^{(i)} \rightarrow 0=0^{(j)} \rightarrow 0=0^{(\operatorname{gcd}(i, j))}$. We now invoke the well-known arithmetical fact that the gcd of any pair $i, j$ is expressible in the form $k i+l j$, where exactly one of the integers $k, l$ is positive. Thus it suffices to prove that $\vdash 0=0^{(i)} \rightarrow 0=0^{(j)} \rightarrow 0=0^{(k i+l j)}$, where $k i+l j$ is positive and only one of $k, l$ is positive. Since we have permutation, $\left(\vdash_{\mathrm{R}} A \rightarrow(B \rightarrow C) \rightarrow B \rightarrow(A \rightarrow C)\right)$, we may assume without loss of generality that it is $k$ which is positive. Now we observe that $\vdash 0=$ $0^{(i)} \rightarrow 0=0^{(j)} \rightarrow 0=0^{(k i)}$. (Reason: (a) $\vdash 0=0^{(j)} \rightarrow 0=0$ follows from $\vdash 0=0^{(j)} \rightarrow 0^{(j)}=0 \rightarrow 0=0$ and $\vdash 0=0^{(j)} \rightarrow 0^{(j)}=0$. (b) $\vdash 0=0 \rightarrow 0=0^{(i)} \rightarrow 0=0^{(i)}$ follows from the transitivity of identity. Combining (a) and (b), $\vdash 0=0^{(j)} \rightarrow 0=0^{(i)} \rightarrow 0=0^{(i)}$. But as already shown, $\vdash 0=0^{(i)} \rightarrow 0=0^{(k i)}$.) But now note that $0=0^{(j)} \rightarrow 0=$ $0^{(k i)} \rightarrow 0=0^{(k i+l j)}$ (Reason: $\vdash 0=0^{(j)} \rightarrow 0^{(k i)} \rightarrow 0^{(j)}=0^{(k i)}$. Since $k i+l j$ is positive and $l<0<k, k i>j$. Therefore, we can write from axiom $\# 1$ - using the proof as in Lemma $5-\vdash 0=0^{(j)} \rightarrow 0=0^{(k i)} \rightarrow$ $0=0^{(k i+(-1) j)}$. Permuting, $\vdash 0=0^{(k i)} \rightarrow .0=0^{(j)} \rightarrow 0=0^{(k i+(-1) j)}$. But now $\vdash 0=0^{(j)} \rightarrow 0=0^{(k i+(-1 j))} \rightarrow 0^{(j)}=0^{(k i+(-1) j)}$. Replacing the last equation by its equivalent, $\vdash 0=0^{(j)} \rightarrow 0=0^{(k i+(-1) j)} \rightarrow 0=0^{(k i+(-2) j)}$. So permuting back, $\vdash 0=0^{(j)} \rightarrow .0=0^{(k i)} \rightarrow 0=0^{(k i+(-2) j)}$. The argument can obviously be repeated to get $\vdash 0=0^{(j)} \rightarrow .0=0^{(k i)} \rightarrow 0=0^{(k i+(-3) j)}$, $\ldots, \vdash 0=0^{(j)} \rightarrow .0=0^{(k i)} \rightarrow 0=0^{(k i+l j)}$ as required.) We can now combine these two using $\vdash_{\mathrm{R}}(A \rightarrow . B \rightarrow C) \rightarrow((B \rightarrow . C \rightarrow D) \rightarrow(A \rightarrow . B \rightarrow D))$, to get the desired result. This proves Proposition 9 .

We also have immediately, that if $i, j$ are relatively prime then $\vdash(0=$ $\left.0^{(i)} \circ 0=0^{(j)}\right) \leftrightarrow 0=0^{\prime}$; and in particular that $\vdash 0=0^{(i)} \rightarrow .0=0^{(j)} \rightarrow$ $0=0^{\prime}$. Now it is possible to show in $\mathrm{RM}^{\sharp}$ that, if $\nvdash 0=0^{(j)} \rightarrow 0=0^{\prime}$, then $\vdash\left(0=0^{(j)} \rightarrow 0=0^{\prime}\right) \rightarrow 0=0^{\prime}$ (see [4] or [5]). We may therefore 
deduce, as promised, that in $\mathrm{RM}^{\sharp}$ if $i$ and $j$ are relatively prime, then one of $\vdash 0=0^{(i)} \rightarrow 0=0^{\prime}$ and $\vdash 0=0^{(j)} \rightarrow 0=0^{\prime}$.

These facts tell a lot of what there is to say about embedding arithmetic of modulus $i$ into the RMn. For example, if $i$ is prime then there are only three points occupied in RMn: $-j$ (at which are $0=0^{\prime}, 0=0^{\prime \prime}, \ldots, 0=0^{(i-1)}$ ), 0 (at which are $0=0,0^{\prime}=0^{\prime}, \ldots, 0^{(k)}=0^{(k)}$, all $k$, and their negations), and $+j$ (the negations of formulae at $-j ;\{-j, 0,+j\}$ are closed in any $\mathrm{RMn}$ with respect to $\&, \forall$ and $\rightarrow$ of course). This is isomorphic to $\mathrm{RM}^{i}$. Furthermore, if $j$ is relatively prime to (any) modulus $i$, then $0=0^{(j)}$ must be equivalent to $0=0^{\prime}$.

It is also immediate that if any two equations $0=0^{(j)}, 0=0^{(k)}$ are at points other than that occupied by $0=0^{\prime}$, then $j, k$ have a common divisor $>1$. Indeed the equation $0=0^{(\operatorname{gcd}(j, k))}$ must also be at some point distinct from $0=0^{\prime}$ (by Proposition 9 again, else one of $0=0^{(j)}, 0=0^{(k)}$ implies $\left.0=0^{\prime}\right)$. Further, the equation $0=0^{(\operatorname{gcd}(j, k))}$ for each pair of points must be no greater than either (else $\vdash 0=0^{(j)} \rightarrow 0=0^{(k j)}$ fails to be verified). Moreover, there is a single common divisor distinct from one for all points distinct from $0=0^{\prime}$ : if there were two, they would have to have a common divisor distinct from $0=0^{\prime}$. And that common divisor is at a point no greater than any other point distinct from $0=0^{\prime}$.

Thus the $\mathrm{RMn}^{i}$ look like this. At the bottom is $0=0^{\prime}, 0=0^{\prime \prime}, \ldots$. The next point up on the Sugihara chain is occupied by a "base" equation $0=0^{(j)}$, and for every other equation $0=0^{(k)}$ higher up the chain (including the modular equation $\left.0=0^{(i)}\right) j$ is a divisor of $k$. If $l$ is a multiple of $k, k$ is no higher than $l$. Every multiple $k$ of $j$ occupies a point distinct from $0=0^{\prime}$, while every nonmultiple of $j$ is equivalent to $0=0^{\prime}$. In addition we have to satisfy $\vdash 0=$ modulus $\rightarrow .0=x \rightarrow 0=$ modulus $-x$, and also $\vdash 0=$ modulus. This means that any two multiples of the base $j$ which add up to the modulus occupy the same point. Where $x$ exceeds the modulus, of course, $0=x$ is equivalent to $0=(x \bmod i)$, where $(x \bmod i)<i$. Equations of the form $x=y$ are equivalent, in virtue of $x=y \leftrightarrow x^{\prime}=y^{\prime}$, to $0=0^{(|(x-y)|)}$. Negations, conjunctions, implications and quantifications are then determined as usual. This does not quite nail down $\mathrm{RMn}^{i}$ uniquely, however. We have for $\mathrm{RM} 7^{16}$, consistent with the above conditions:

Australasian Journal of Logic (18:5) 2021, Article no. 6 

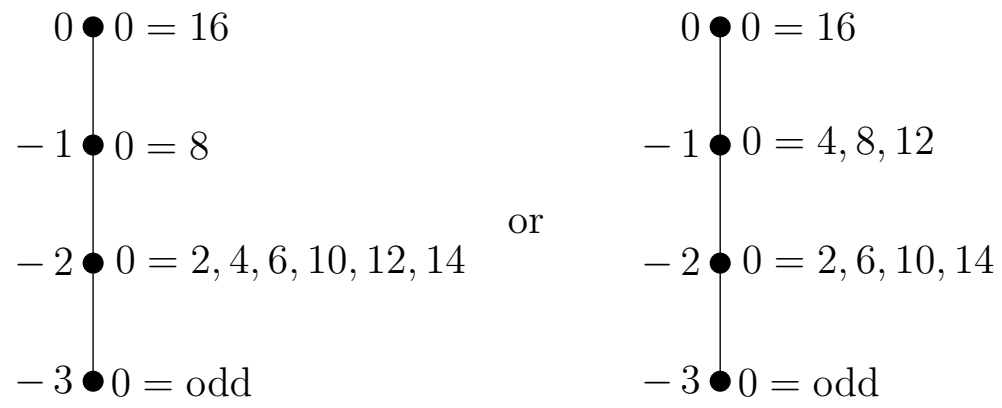

but there do not seem to be any further conditions restricting the $\mathrm{RMn}^{i}$. That is, it seems to be that any $\mathrm{RMn}^{i}$ satisfying the above constraints is possible. In particular, for any $i$ there is some $n$ (e.g. 3) satisfying the constraints; and for any (odd) $n$ there is some $i$ (if $n=2 j+1$, take e.g. arithmetic modulus $2^{j}$, and for $k \leq j$, send $0=0^{(2 j-k)}$ to point $-k$ and make appropriate adjustments).

Thus modulus and size of matrix interact strongly, and not all combinations resist isomorphism with a smaller matrix size. We will however continue to refer in the next section to $\mathrm{RMn}^{i}$, with the understanding that these may not be unique and that a particular $i$ might force an isomorphism with $\mathrm{RMm}^{i}$ for lesser $m$. With that qualification, we note that the $\mathrm{RMn}^{i}$ all have the properties of the $\mathrm{RM}^{i}$ : inconsistency, nontriviality, containment of $\mathrm{RM}^{\sharp \sharp}$ etc., completeness, primeness, $\omega$-completeness, $\omega$-inconsistency. We omit the proofs.

\section{The Arithmetic $\mathrm{RM}^{\omega}$}

In this section we study the intersection of all the models $\mathrm{RMn}^{i}$.

Definition 6. The arithmetic $\mathrm{RM}^{\omega}$ is the intersection of all the truths of all the $\mathrm{RMn}^{i}$.

Proposition 10. $\mathrm{RM}^{\omega}$ is inconsistent, $\omega$-inconsistent and nontrivial. If $n$ and $m$ are distinct, then $0^{(n)}=0^{(m)}$ is not in $\mathrm{RM}^{\omega}$.

Proof. Every $\mathrm{RMn}^{i}$ contains $0=0, \neg 0=0$. Also, every $\mathrm{RMn}^{i}$ contains $\neg 0=0, \neg 0=0^{\prime}, \neg 0=0^{\prime \prime}, \ldots$ but also $\neg(x) \neg 0=x$. Further, $0=0^{\prime}$ is not in some (in fact all) $\mathrm{RMn}^{i}$. Finally, $0^{(n)}=0^{(m)}$ is not in $\mathrm{RM}^{|n-m|+1}$. 
Proposition 11. $\mathrm{RM}^{\omega}$ is $\omega$-complete.

Proof. Let $F 0, F 0^{\prime}, F 0^{\prime \prime}, \ldots$ all be in $\mathrm{RM}^{\omega}$. Then they are true in every $\mathrm{RMn}^{i}$. But every $\mathrm{RMn}^{i}$ is $\omega$-complete, so $(x) F x$ is true in every $\mathrm{RMn}^{i}$, and so is in $\mathrm{RM}^{\omega}$.

Proposition 12. $\mathrm{R}^{\sharp}, \mathrm{R}^{\sharp}, \mathrm{RM}^{\sharp}, \mathrm{RM}^{\sharp \sharp}$ are all included in $\mathrm{RM}^{\omega}$.

Proof. Each of these systems is included in every $\mathrm{RMn}^{i}$.

Proposition 13. The nontheorems of $\mathrm{RM}^{\omega}$ are recursively enumerable.

Proof. This is a standard type of argument. Every $\mathrm{RMn}^{i}$ is decidable, so set the Turing machines for each $\mathrm{RMn}^{i}$ to work consecutively. Eventually, if $A$ is a nontheorem of some $\mathrm{RMn}^{i}$, some Turing machine will say so.

Problem 1. Is $\mathrm{RM}^{\omega}$ decidable? Is it axiomatisable? Say by adding $\neg(x) \neg 0=$ $x^{\prime}$ to $\mathrm{RM}^{\sharp}$ ?

Proposition 14. $\mathrm{RM}^{\omega}$ is incomplete.

Proof. Consider the wff $A: 0=0^{(2)} \rightarrow 0=0^{\prime}$. In $\mathrm{RM}^{2}$, this gets the value $0 \rightarrow-1=-1$, and so is not a truth. In $\mathrm{RM}^{3}$, however, it gets $-1 \rightarrow-1=+1$; and so its negation, $\neg\left(0=0^{(2)} \rightarrow 0=0^{\prime}\right)$ gets value -1 and so is not a truth of $\mathrm{RM}^{3}$. Hence, neither $A$ nor $\neg A$ is in $\mathrm{RM}^{\omega}$.

Thus we also have:

Proposition 15. $\mathrm{R}^{\sharp}, \mathrm{R}^{\sharp}, \mathrm{RM}^{\sharp}, \mathrm{RM}^{\sharp \sharp}$ are all incomplete.

If Proposition 1 sidesteps Gödel's second incompleteness theorem, the significance of these last two propositions is that they parallel and contrast with - though very simply - Gödel's first incompleteness theorem. They are not conditional, as the Gödel/Rosser theorem is, on the assumption of consistency; indeed $\mathrm{RM}^{\omega}$ is inconsistent. Nor do they need methods not formalisable in $\mathrm{P}^{\sharp}$ to prove the assumption of consistency and so detach the conclusion of incompleteness: the above proof is perfectly finitistic. In addition, the present result holds independently of the assumption of a recursively enumerable proof procedure, unlike the Gödel/Rosser theorem. Any non-recursively-enumerable arithmetic in the language $\mathscr{L}$ and included in $\mathrm{RM}^{\omega}$ has the same property. In particular, $\mathrm{R}^{\sharp}$ and $\mathrm{RM}^{\sharp \sharp}$ are not recursively enumerable.

Australasian Journal of Logic (18:5) 2021, Article no. 6 
The case for some relevant arithmetic being true arithmetic rests on the claim that the deductive relations of relevant logic are correct while those of classical logic admit invalid inferences. Quantified R looks to be a good candidate for the logic in question (though the present remarks apply to any sublogic of $R$ ). The objection to $R^{\sharp}$ as being true arithmetic, however, is just the objection to $P^{\sharp}$ : since it is recursively enumerable and since all primitive recursive functions are representable therein, if consistent it is incomplete. The move to $P^{\sharp \sharp}$ as a candidate is mirrored relevantly by the move to $R^{\sharp \sharp}$. But unlike the case of $P^{\sharp \sharp}$, the expanded language of $R^{\sharp \sharp}$ means that we do not have a guarantee of completeness via the $\Omega$-rule. The above incompleteness results mean that $R^{\sharp \sharp ~(a n d ~} R M^{\sharp \sharp}$ ) are open to the same objection that $P^{\sharp}$ and $R^{\sharp}$ are. Mind you, the mere fact of incompleteness in a candidate for true arithmetic does not seem to us to be so serious (see e.g. Mortensen and Priest [9]). More serious is what is unprovable: neither $0=2 \rightarrow 0=1$ nor $\neg(0=2 \rightarrow 0=1)$. For what our intuition is worth, the latter feels true to us.

The fact that $\mathrm{RM}^{\omega}$ is incomplete implies that the $\mathrm{RMn}^{i}$ do not form a chain or even a (lower) semilattice under subsethood. For if they did, it would be simple to show that since all the $\mathrm{RMn}^{i}$ are complete, so must $\mathrm{RM}^{\omega}$ be. However, $\mathrm{RM}^{\omega}$ is complete in a weaker sense. To show this, it is instructive to take a detour through the $\mathrm{RMn}^{i}$.

Definition 7. $\mathrm{RM}^{\omega}=\bigcap_{i} \mathrm{RM}^{i}$.

Definition 8. A wff of $\mathscr{L}$ is extensional iff it contains no occurrences of $\rightarrow$. A theory $T h$ is extensionally complete iff for every closed extensional wff $A$, either $A \in T h$ or $\neg A \in T h$.

Proposition 16. If $A$ is a closed extensional wff, then, for any $k$ and any $i$, if $A$ is true in $\mathrm{RM}^{k i}$ then $A$ is true in $\mathrm{RM}^{i}$.

Proof. We use the fact that in all these arithmetics $\neg(A \& B) \leftrightarrow \neg A \vee \neg B$ and $\neg \forall=\exists \neg$, to drive negations through conjunctions and quantifiers. So we only need to consider wffs which are disjunctions, conjunctions and universal and existential quantifications of basic equations and unequations (i.e. of the form $\neg t_{1}=t_{2}$ ). The quantifiers can also be eliminated in favour of finite conjunctions and disjunctions in the usual way, so we only need to consider conjunctions and disjunctions of basic equations and unequations. Further, for the latter we need only consider the terms in $\left\{0,0^{\prime}, \ldots, 0^{(k i-1)}\right\}$. 
Base. If $0^{(n)}=0^{(m)}$ is true in $\mathrm{RM}^{k i}$, then for some $j \geq 1,|n-m|=j k i$. Hence $|n-m|=(j k) i$, where $j k \geq 1$; so $0^{(n)}=0^{(m)}$ takes value 0 in $\mathrm{RM}^{i}$. No equation takes the value +1 in $\mathrm{RM}^{k i}$. Equally, however, no unequation takes the value -1 in any $\mathrm{RM}^{l}$; so trivially every unequation true in $\mathrm{RM}^{k i}$ is true in $\mathrm{RM}^{i}$.

Inductive step. (i) $A$ is $B \& C$ and true in $\mathrm{RM}^{k i}$. Then $B$ and $C$ are both true in $\mathrm{RM}^{k i}$, whence by the inductive hypothesis they are both true in $\mathrm{RM}^{i}$, so $B \& C$ is. (ii) $A$ is $B \vee C$ and true in $\mathrm{RM}^{k i}$. But $\mathrm{RM}^{k i}$ is prime, so at least one of $B, C$ is true in $\mathrm{RM}^{k i}$, so by the inductive hypothesis is true in $\mathrm{RM}^{i}$, so $B \vee C$ is. This completes the proof.

Proposition 17. The extensional truths of the $\mathrm{RM}^{i}$ form a lower semilattice with respect to subset inclusion.

Proof. Any pair of arithmetics $\mathrm{RM}^{i}, \mathrm{RM}^{j}$ have a common lower bound $\mathrm{RM}^{i j}$, by the previous proposition. A greatest lower bound is then available since there are only a finite number of candidates

We note that the lower semilattice is not complete (as a semilattice) since the infinite set $\left\{\mathrm{RM}^{i}: i\right.$ prime $\}$ has no lower bound in the semilattice. (We can complete it, however, by adding the extensional truths of RM3 ${ }^{\omega}$.) The previous proposition now enables us to prove:

Proposition 18. RM3 ${ }^{\omega}$ is extensionally complete.

Proof. If $A$ is extensional and not true in $\mathrm{RM}^{\omega}$ it is not true in $\mathrm{RM}^{i}$ for some $i$. If $\neg A$ is not true in $\mathrm{RM}^{\omega}$ it is not true in $\mathrm{RM}^{j}$ for some $j$. Hence neither $A$ nor $\neg A$ would be true in $\mathrm{RM}^{i j}$. But every $\mathrm{RM}^{k}$ is complete, contradiction.

Now we can return to the question of the extensional completeness of $\mathrm{RM}^{\omega}$.

Proposition 19. For each $i$ and $n$ the extensional truths of $\mathrm{RM}^{i}$ are just those of $\mathrm{RMn}^{i}$.

Proof. We prove that $\mathrm{RM}^{i} \subseteq \mathrm{RMn}^{i}$; the converse is similar. As before, we take the base clause to equations and unequations and the induction with respect to \&, $\vee, \forall, \exists$; and we ignore terms other than $\left\{0, \ldots, 0^{(i-1)}\right\}$ because of $\vdash(x)\left(x=0 \vee \ldots \vee x=0^{(i-1)}\right)$. Further, we can ignore the $\forall, \exists$ cases, since in 
both $\mathrm{RM}^{i}$ and $\mathrm{RMn}^{i}, \forall$ and $\exists$ are eliminable in favour of \& and $\vee$. Evidently, the equations of $\mathrm{RM}^{i}$ are just those of the form $0^{(n)}=0^{(n+k i)}$ for some $k \geq 0$. All of these are true in $\mathrm{RMn}^{i}$. Also, every unequation (i.e. $\neg 0^{(n)}=0^{(m)}$ ) is in $\mathrm{RMn}^{i}$ anyway. The inductive clause for $\&$ is straightforward. For $\vee$, we use the fact that $\mathrm{RM}^{i}$ is prime.

Now, however, we can observe that it follows from Proposition 19 that the extensional truths of $\mathrm{RM}^{\omega}$ are exactly those of $\mathrm{RM}^{\omega}{ }^{\omega}$. (Reason: $\mathrm{RM}^{\omega} \subseteq \mathrm{RM} 3^{\omega}$ is evident. Conversely, if $A \in \mathrm{RM}^{\omega}$ then $A \in \mathrm{RM}^{i}$ for all $i$; so by Proposition $19, A \in \mathrm{RMn}^{\omega}$ for $i$.) It immediately follows that

Proposition 20. $\mathrm{RM}^{\omega}$ is extensionally complete.

The route we chose to Proposition 20 has the interest that it reveals the relations between the $\mathrm{RM}^{i}$ and on the one hand $\mathrm{RM}^{k i}$ and on the other $\mathrm{RMn}^{i}$. However, Proposition 20 also follows from a more general, though perhaps less deep, result, Proposition 21.

Definition 9. A secondary equation is any sentence $A$ such that $\vdash A \rightarrow \mathbf{t}$. A secondary unequation is any sentence $A$ such that $\vdash \mathbf{f} \rightarrow A$ (see [4] or [5]; recall from Definition 5 that $\mathbf{t}$ is $0=0$ and $\mathbf{f}$ is $\neg 0=0$ ).

Proposition 21. Any inconsistent arithmetic extending $\mathrm{R}^{\sharp}$ is extensionally complete.

Proof. The details are contained in [4] or [5]. First note that in any inconsistent extension of $\mathrm{R}^{\sharp}, \vdash \mathbf{t} \rightarrow \mathbf{f}$ (See [4] or [5]; essentially the reason is that if $\vdash A \& \neg A$ for some $A$, then since $\vdash \neg A \leftrightarrow(A \rightarrow \mathbf{f}), \vdash \mathbf{f}$. But $\vdash \mathbf{f} \leftrightarrow(\mathbf{t} \rightarrow \mathbf{f})$, so $\vdash \mathbf{t} \rightarrow \mathbf{f}$.) But it can be shown that union of the secondary equations and secondary unequations is closed with respect to the operators $\neg, \&$ and $\forall$. Finally it can be shown that every equation is a secondary equation and every unequation a secondary unequation. Proposition 21 follows.

One final point to make is to show again how, relevantly, concepts can diverge which coincide classically.

Definition 10. A theory is E-complete iff whenever $(\exists x) F x$ is in the theory, so is [one of $F 0$ ], $F 0^{\prime}, F 0^{(2)}, \ldots$.

[In $\mathrm{RM}^{\omega}$, we may expect E-completeness] with respect to extensional wffs to coincide with $\omega$-completeness. However, we have, despite the $\omega$-completeness of $\mathrm{RM}^{\omega}$ :

Australasian Journal of Logic (18:5) 2021, Article no. 6 
Proposition 22. There is an extensional wff $(\exists x) F x$ in $\mathrm{RM}^{\omega}$ such that none of $F 0, F 0^{\prime}, F 0^{(2)}, \ldots$ are in $\mathrm{RM}^{\omega}$.

Proof. $(\exists x)\left(0=x^{\prime}\right)$ is in $\mathrm{RM}^{\omega}$ since it is in every $\mathrm{RMn}^{i}$. But $0=0^{\prime}, 0=0^{\prime \prime}$, ... are each refutable in some $\mathrm{RMn}^{i}$, and so not in $\mathrm{RM}^{\omega}$.

The explanation of the difference from the classical case is, of course, that classically a theory is either consistent or trivial, and consistency together with completeness guarantees E-completeness iff $\omega$-completeness no matter what the logic in question. But relevantly, theories can be neither consistent and nontrivial. The equivalence can thus break down in such cases.

\section{An Inconsistent Extension of $R^{\sharp}$ Without Mingle}

The finite arithmetics studied up to now have all had the property that mingle, $A \rightarrow . A \rightarrow A$ holds, so that one of $A \rightarrow B, B \rightarrow A$ is true. In the present section we display an arithmetic for which this is not so. For base logic we take the ten-valued lattice, which we call CL2, with Hasse diagram as follows: ${ }^{3}$

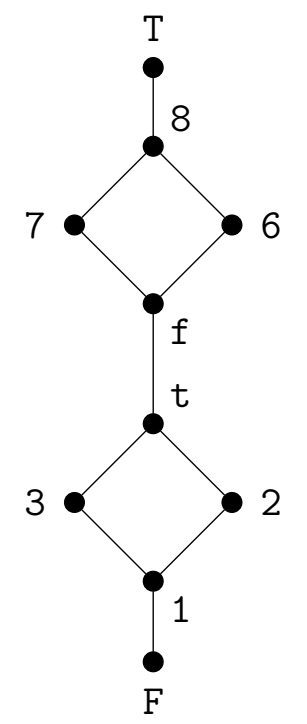

\footnotetext{
${ }^{3}$ We acknowledge in this section the help of John Slaney's computer programs, and the DEC-10 computer for executing them.
}

Australasian Journal of Logic (18:5) 2021, Article no. 6 
Negation is as suggested by the notation: $\mathrm{F}=\neg \mathrm{T}, 1=\neg 8,2=\neg 7,3=\neg 6$ and $t=\neg f$ All values above and including $t$ are designated. Conjunctions and disjunctions are respectively lower and upper bounds in the lattice. The $\rightarrow$ table is as follows.

\begin{tabular}{ll|llllllllll} 
& $\rightarrow$ & $\mathrm{F}$ & 1 & 2 & 3 & $\mathrm{t}$ & $\mathrm{f}$ & 6 & 7 & 8 & $\mathrm{~T}$ \\
\hline & $\mathrm{F}$ & $\mathrm{T}$ & $\mathrm{T}$ & $\mathrm{T}$ & $\mathrm{T}$ & $\mathrm{T}$ & $\mathrm{T}$ & $\mathrm{T}$ & $\mathrm{T}$ & $\mathrm{T}$ & $\mathrm{T}$ \\
& 1 & $\mathrm{~F}$ & $\mathrm{t}$ & $\mathrm{t}$ & $\mathrm{t}$ & $\mathrm{t}$ & 8 & 8 & 8 & 8 & $\mathrm{~T}$ \\
& 2 & $\mathrm{~F}$ & 3 & $\mathrm{t}$ & 3 & $\mathrm{t}$ & 7 & 8 & 7 & 8 & $\mathrm{~T}$ \\
& 3 & $\mathrm{~F}$ & 2 & 2 & $\mathrm{t}$ & $\mathrm{t}$ & 6 & 6 & 8 & 8 & $\mathrm{~T}$ \\
$*$ & $\mathrm{t}$ & $\mathrm{F}$ & 1 & 2 & 3 & $\mathrm{t}$ & $\mathrm{f}$ & 6 & 7 & 8 & $\mathrm{~T}$ \\
$*$ & $\mathrm{f}$ & $\mathrm{F}$ & $\mathrm{F}$ & $\mathrm{F}$ & $\mathrm{F}$ & $\mathrm{F}$ & $\mathrm{t}$ & $\mathrm{t}$ & $\mathrm{t}$ & $\mathrm{t}$ & $\mathrm{T}$ \\
$*$ & 6 & $\mathrm{~F}$ & $\mathrm{~F}$ & $\mathrm{~F}$ & $\mathrm{~F}$ & $\mathrm{~F}$ & 3 & $\mathrm{t}$ & 3 & $\mathrm{t}$ & $\mathrm{T}$ \\
$*$ & 7 & $\mathrm{~F}$ & $\mathrm{~F}$ & $\mathrm{~F}$ & $\mathrm{~F}$ & $\mathrm{~F}$ & 2 & 2 & $\mathrm{t}$ & $\mathrm{t}$ & $\mathrm{T}$ \\
$*$ & 8 & $\mathrm{~F}$ & $\mathrm{~F}$ & $\mathrm{~F}$ & $\mathrm{~F}$ & $\mathrm{~F}$ & 1 & 2 & 3 & $\mathrm{t}$ & $\mathrm{T}$ \\
$*$ & $\mathrm{~T}$ & $\mathrm{~F}$ & $\mathrm{~F}$ & $\mathrm{~F}$ & $\mathrm{~F}$ & $\mathrm{~F}$ & $\mathrm{~F}$ & $\mathrm{~F}$ & $\mathrm{~F}$ & $\mathrm{~F}$ & $\mathrm{~T}$
\end{tabular}

It is mechanical (literally!) to verify that all the theorems of $\mathrm{R}$ hold in CL2. Note though that mingle fails e.g. at $7 \rightarrow .7 \rightarrow 7$. Into CL2 we code arithmetic modulo 105 , which we call $\mathrm{CL}^{105}$, via the following rules.

(1) $I\left(0^{(n)}=0^{(m)}\right)=\mathrm{t}$ iff for some $k \geq 0,|n-m|=105 k$

(2) $\quad I\left(0^{(n)}=0^{(m)}\right)=3$ iff for some $k \geq 1,|n-m|=15 k$ but for no $k \geq 1,|n-m|=21 k$

(3) $\quad I\left(0^{(n)}=0^{(m)}\right)=2$ iff for some $k \geq 1,|n-m|=21 k$ but for no $k \geq 1,|n-m|=15 k$

(4) $\quad I\left(0^{(n)}=0^{(m)}\right)=1$ iff for some $k \geq 1,|n-m|=3 k$ but for no $k \geq 1,|n-m|=15 k$ and for no $k \geq 1,|n-m|=21 k$

(5) $\quad I\left(0^{(n)}=0^{(m)}\right)=\mathrm{F}$ otherwise, i.e. iff for no $k \geq 1,|n-m|=3 k$

Negations, conjunctions and $\rightarrow$ are as for CL2. Quantifiers are now treated as they were in $\S 2$, and that enables us to verify the quantificational axioms of $\mathrm{R}$ as well.

Proposition 23. The theorems of $\mathrm{R}^{\sharp \sharp}$ are all true in $\mathrm{CL} 2^{105}$.

Proof. The details are omitted. Axiom \#2 is lengthy, but not in principle difficult. 
Problem 2. What are the general conditions under which a modular arithmetic can be embedded in a nonmingle lattice? What mods will admit of such treatment (not all do, which explains the otherwise bizarre choice of modulo 105), and what conditions must equations sent to incomparable points satisfy?

\section{Fermat's Last Theorem}

One advantage of having inconsistent extensions of $P^{\sharp}, R^{\sharp}$, etc., is that we can entertain the possibility of constructing inconsistent models to falsify arithmetical sentences, the truth of which is problematic. Take, for example, Fermat's last theorem. As is well known, if the denial of FLT is not provable in arithmetic then FLT is true. Hence if in some inconsistent model of $\mathrm{P}^{\sharp}$ $\neg$ FLT can be made to fail, FLT must be true. Unfortunately, in RM $^{\omega}$ and all its finite modular extensions, $\neg$ FLT is equivalent to FLT (considering both as extensional formulae), and both are true. In fact the inability to refute $\neg$ FLT is not confined to an RM-ish logic. Given any model in which $\vdash \mathbf{t} \rightarrow \mathbf{f}$, i.e. in any inconsistent extension of $\mathrm{R}^{\sharp}$, it can be shown that $\neg$ FLT $\rightarrow$ FLT, but that both $\neg$ FLT and FLT are true (though not necessarily equivalent). Of course, this does not rule out the possibility that there are other ways of producing inconsistent extensions of $\mathrm{P}^{\sharp}$ which would yield a refutation of $\neg$ FLT. We remark, for what it is worth, that the intensional version of FLT, i.e. $(x, y, z, n)\left(x^{n}+y^{n}=z^{n} \rightarrow x=0 \vee x=1 \vee y=0 \vee y=1 \vee z=0 \vee z=\right.$ $1 \vee n=0 \vee n=1 \vee n=2$ ), is refutable. Choose $x=y=z=n=3$ in $\mathrm{RM}^{9}$. Then for this instance, the consequent takes value -1 . But the antecedent takes the value of $18=9$ which in $\mathrm{RM}^{9}$ is 0 . But $0 \rightarrow-1=-1$, and the value of a universal quantification is the minimum of the values of its instances. So the value of the intensional FLT is -1 (and so it is not provable in $R^{\sharp}$ ).

\section{Conclusion}

We conclude with the observation that, since negation consistent theories are a special case of nontrivial theories, there is no reason for relevant mathematics to "reject" model theory as classically conceived. Relevant mathematics proposes itself as an extension to the classical case rather than as an alternative, though it must be admitted that the extension might be seen

Australasian Journal of Logic (18:5) 2021, Article no. 6 
to have more desirable properties by being less restrictive. An example is the classical identification of negation consistency with nontriviality, which has, we believe, no justification. Relaxation of that identification means, for example, that limitative theorems such as those of Gödel have to be re-examined. It also means, as we have seen, that theories can be considered in which concepts which coincide classically no longer coincide, but are nonetheless mathematically well-behaved and interesting. Furthermore, it opens the possibility that inconsistent arithmetics might enable the solution of traditional problems via the demonstration of the independence of problematic sentences from formalised arithmetic; the latter being, of course, a traditional hope of model theory. But we stress that models developed classically do not suddenly fail to be legitimate in relevant mathematics. Instead, one can choose to vary the class of truths in the model in accordance with some nonclassical logic, and compare the result with the classical case. The resulting change in viewpoint cannot impoverish insight into the nature of mathematical structures, but rather can only enrich it.

\section{Authors' Acknowledgements}

We wish to thank Graham Priest and Paul Thistlewaite for their help.

\section{Editors' Acknowledgments}

This paper was previously published as:

- R. K. Meyer and C. Mortensen. Inconsistent models for relevant arithmetics. Journal of Symbolic Logic, 49(3):917-929, 1984.

It appears courtesy of the Association for Symbolic Logic, who retain the copyright to the paper.

\section{References}

[1] A. R. Anderson and N. D. Belnap. Entailment, volume I. Princeton University Press, Princeton, 1975.

Australasian Journal of Logic (18:5) 2021, Article no. 6 
[2] J. M. Dunn. Quantum mathematics. volume 2, pages 512-531, East Lansing, Michigan, 1981. Philosophy of Science Association.

[3] R. K. Meyer. Relevant arithmetic. Bulletin of the Section of Logic, 5:133-137, 1976.

[4] R. K. Meyer. The consistency of arithmetic. Unpublished monograph, 1976.

[5] R. K. Meyer. Arithmetic formulated relevantly. Unpublished monograph, 1976.

[6] R. K. Meyer. $P^{\sharp \#}$ is included in $R^{\sharp \#}$. Unpublished monograph, 1976.

[7] R. K. Meyer. $\mathrm{R}_{I^{-}}$The bounds of finitude. Zeitschrift für Mathematische Logik und Grundlagen der Mathematik, 14:385-387, 1970.

[8] C. Mortensen. Model structures and set algebras for Sugihara matrices. Notre Dame Journal of Formal Logic, 23(1):85-90, 1982.

[9] C. Mortensen and G. Priest. The truth teller paradox. Logique et Analyse, 24(95-96):381-388, 1981.

[10] R. Routley. Ultralogic as universal. pages 892-962. Philosophy Department, Research School of Social Sciences, Australian National University, Canberra, 1980.

[11] F. G. Asenjo. Towards an antinomic mathematics. pages 394-412. Philosophia Verlag, München, 1989.

Australasian Journal of Logic (18:5) 2021, Article no. 6 